\title{
Lupus Nephritis with Visual Field Defect Secondary to Hypertensive Retinopathy: A Case Report
}

\author{
Hor SM, Norshamsiah MD ( $₫)$, Mushawiahti M, Hazlita MI \\ Department of Ophthalmology, Faculty of Medicine, Universiti Kebangsaan Malaysia Medical Centre, \\ Jalan Yaacob Latif, Bandar Tun Razak, 56000 Cheras, Kuala Lumpur, Malaysia.
}

\begin{abstract}
A 23-year-old lady presented with both eye progressive painless blurring of vision for two weeks in 2011. Prior to that she had malar rash, hair loss, photosensitivity and bilateral leg swelling. Ocular examination showed that visual acuity on the right was $6 / 60$ and on the left was $6 / 24$. Both optic disc were swollen with extensive peripapillary cotton wool spot (CWS), flame shape haemorrhages, dilated and tortuous vessels with macular oedema. Systemic examination revealed blood pressure of 176/111 mmHg, malar rash and alopecia. Diagnosis of grade 4 hypertensive retinopathy secondary to SLE was made. The diagnosis was confirmed by positive ANA/ dsDNA, low C3/ C4 and renal biopsy showed lupus nephritis. She was treated with oral prednisolone, hydroxychloroquine and cyclosporin A. Throughout the monitoring for hydroxychloroquine toxicity, vision over both eyes were $6 / 9$, but serial visual fields showed non-progressive left superior and inferior scotoma while right eye showed inferior scotoma. The intraocular pressure was normal with pink optic disc and cup disc ratio of 0.3. Optical coherence tomography (OCT) showed temporal and nasal retinal nerve fiber layer thinning bilaterally. However, macula OCT, fundus fluorescein angiography and autofluorescence were normal. The visual field defect was concluded secondary to CWS indicating microinfarction of the retinal nerve fiber secondary to previous hypertensive retinopathy. Non-progressive visual field defects may occur after the appearance of CWS in hypertensive retinopathy and it should not be overlooked when diagnosing glaucoma or hydroxychloroquine toxicity.
\end{abstract}

Keywords: $\quad$ hydroxychloroquine, hypertensive retinopathy, lupus nephritis, scotoma, visual fields

\section{Correspondence:}

Norshamsiah Md Din. Department of Ophthalmology, Faculty of Medicine, Universiti Kebangsaan Malaysia Medical Centre, Jalan Yaacob Latif, Bandar Tun Razak, 56000 Cheras, Kuala Lumpur, Malaysia. Tel: +603-91457207 Fax: +603-91456733 Email: shamsiahdr@hotmail.com

Date of submission: 15 Aug, 2017

Date of acceptance: 10 Sept, 2017

\section{Introduction}

Systemic lupus erythematosus (SLE) is an autoimmune disease with a wide range of clinical manifestation which can affect any organ in the body (1). Secondary malignant hypertension resulting from renal parenchymal lesion is part of the disease entity. Malignant hypertension may affect the eye in various ways but grade III or IV hypertensive retinopathy with ischemic retinal injury can be visually disabling. Cotton wool spots seen in grade III and IV hypertensive retinopathy indicates microinfarction of the retinal nerve fibers and neuronal loss which can results in subsequent related scotoma (2). Hereby, we describe a case of lupus nephritis with grade 4 hypertensive retinopathy presenting with multiple peripapillary cotton wool spots which eventually causes permanent visual field defect.

\section{Case Report}

A 23-year-old lady presented with both eye progressive painless blurring of vision for two weeks in 2011. Prior to that she had malar rash, hair loss, photosensitivity and bilateral leg swelling. The past medical and family history were insignificant. 


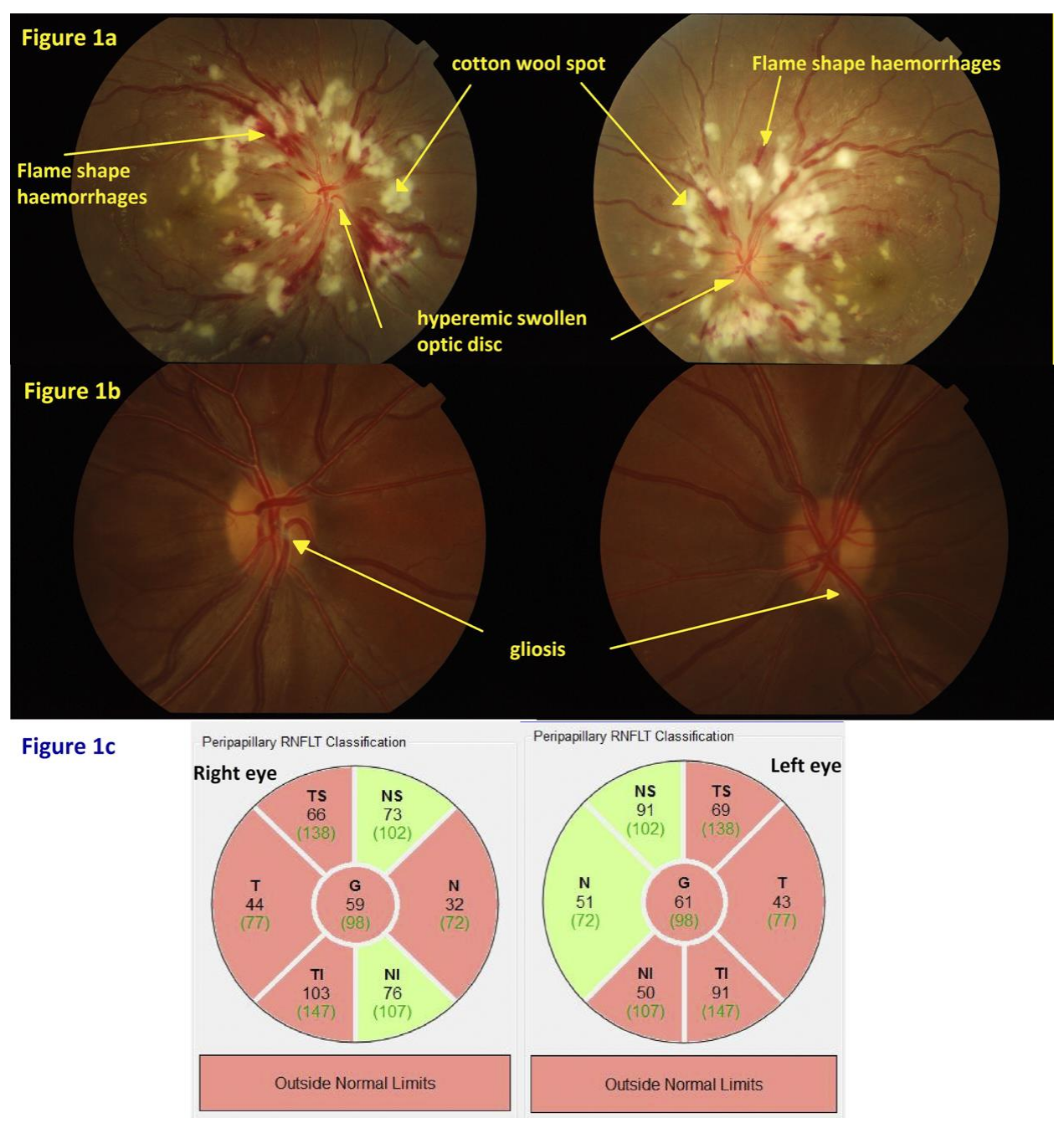

Figure 1: a) Optic disc photo taken in year 2011 showed hypertensive retinopathy as evidenced by hyperemic swollen disc, extensive peripapillary cotton wool spots and flame shape haemorrhages; b) Optic disc photo taken in 2016 showed pink optic disc with cup/disc ratio 0.3 , no neuroretinal rim thinning, scant gliosis more on right eye; c) OCT RNFL showed thinning of bilateral temporal quadrant, right nasal quadrant and left infero-nasal quadrant.

Ocular examination showed that visual acuity on the right was $6 / 60$ and left $6 / 24$. There was no relative afferent pupillary defect. Anterior segment and intraocular pressure were normal. Fundoscopic examination showed bilateral optic disc swelling with blurred disc margin, extensive peripapillary cotton wool spot (CWS), flame shape haemorrhages, dilated and tortous vessels and macular oedema (Fig. 1a). Systemic examination showed blood pressure of $176 / 111 \mathrm{mmHg}$, malar rash, discoid rash over the scalp, alopecia and vasculitic lesions over her palms.

\section{The blood investigation showed positive} ANA/dsDNA, low C3/C4, macrocytic anemia with positive direct coomb's test and raised reticulocyte count. Urinalysis showed proteinuria with microscopic haematuria and renal biopsy revealed diffuse active proliferative and membranous lupus nephritis (WHO class IV \& V) with $56 \%$ cellular crescent. Otherwise, the renal function test was normal and computed tomography (CT) of the brain did not show features of posterior reversible encephalopathy syndrome (PRES). A diagnosis of SLE with grade IV hypertensive retinopathy secondary to lupus nephritis, autoimmune hemolytic anemia and discoid lupus was made. She was treated with intravenous (IV) methylprednisolone, double-filtration plamapheresis with IV immunoglobulin and IV cyclophosphamide. Subsequently, the prednisolone was tapered off and she was maintained on HCQ 200mg daily.

She was seen in Ophthalmology clinic for screening of hydroxychloroquine toxicity. Throughout the follow up, her visual acuity remained stable at 6/9 in both eyes 

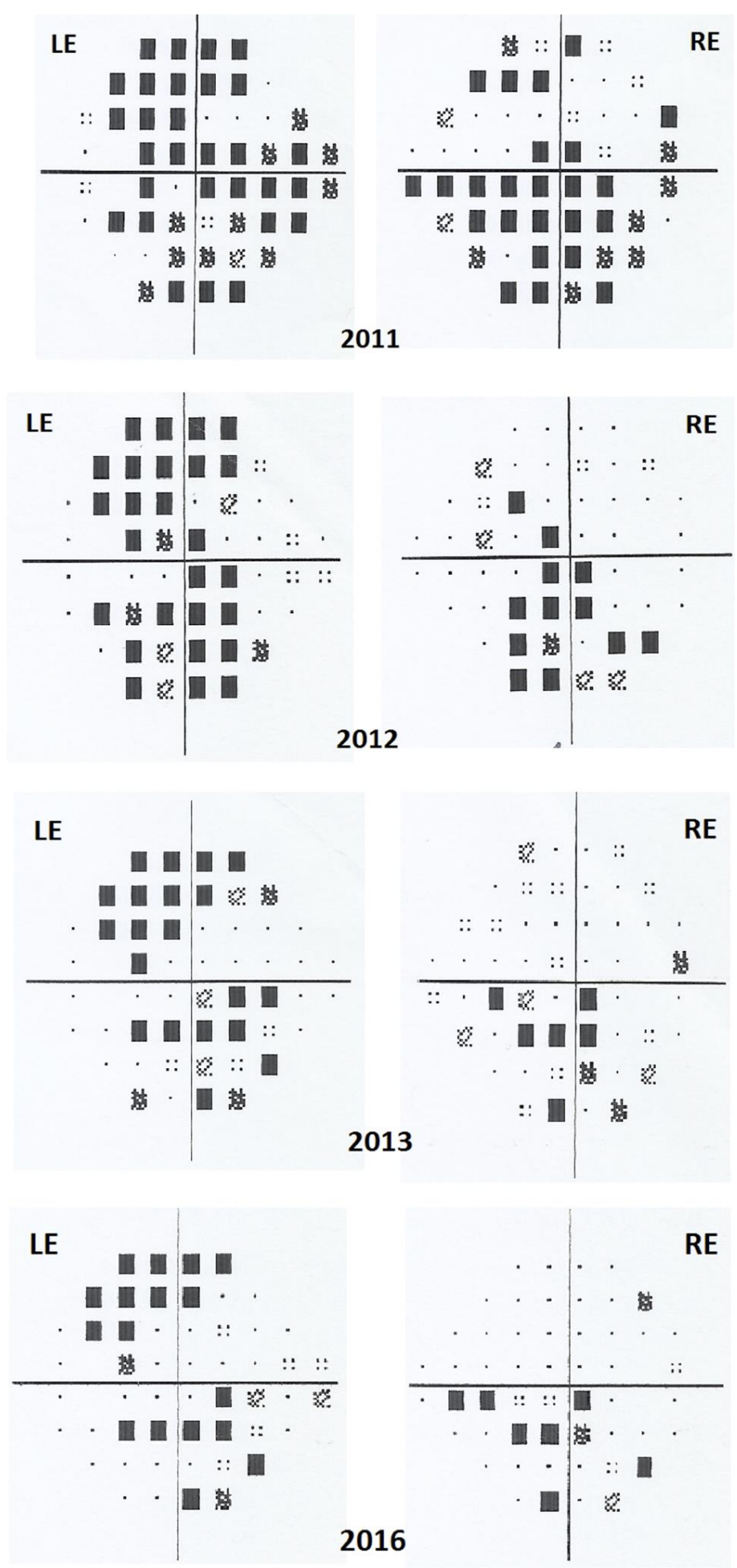

Figure 2: Serial visual field showed persistent left superior and inferior arcuate scotoma and right inferior arcuate scotoma with lesser density over time. (RE, right eye; LE, left eye)

with normal intraocular pressure. The optic disc was pink, cup to disc ratio was 0.3 with no neuroretinal rim thinning (Fig. 1b). However, serial visual fields showed left superior and inferior arcuate scotoma and right inferior field defect which was getting less dense. The latest visual field showed left superior arcuate and inferior paracentral scotoma while the right had some residual inferior paracentral scotoma (Fig. 2). The 
color vision test was normal. A possibility of HCQ toxicity was ruled out as the fundus fluorescein angiography (FFA), fundus autofluorescence (FAF) and optical coherence tomography (OCT) macular were normal. OCT of retinal nerve fiber layer (RNFL) however showed thinning in both eyes (Fig. 1c). The visual field defect was concluded secondary to CWS indicating microinfarction of the retinal nerve fiber secondary to previous hypertensive retinopathy due to lupus nephritis.

\section{Discussion}

SLE is a multisystem, autoimmune, connective-tissue disorder with various clinical presentation and follows a relapsing and remitting course (1). The management often depends on disease severity and clinical manifestation. Medications include antimalarials (e.g. HCQ), nonsteroidal anti-inflammatory drugs (NSAIDS), steroids, non-biologic and biologic disease-modifying antirheumatic drugs (DMARDs). HCQ is important for long-term treatment of SLE as LUMINA (Lupus in Minorities: Nature versus Nurture) study and other trials have shown a decrease in flares and prolonged life in patients treated with HCQ (3).

However, HCQ has been known to cause retinal toxicity for many years and ophthalmology screening is necessary. The mechanism of retinal toxicity though is not well understood but the primary damage is to the photoreceptors followed by disruption of the RPE (4). HCQ retinopathy is irreversible. Thus, early detection of retinopathy is important before RPE damage as there is only limited progression after stopping the medication and the fovea is not threatened (5). Recommended screening tests are automated visual fields and spectral-domain OCT macular (6). Our patient was noted to have non-progressive arcuate scotoma in both eyes by serial visual fields in contrast to the progressive nature of visual field defect seen in HCQ retinopathy. Furthermore, the macular OCT did not show loss of outer nuclear layer or RPE disruption and the FAF did not show increased fluorescence at the parafoveal or over the macular region. Neither did FFA showed any hyperfluorescence lesion which represents RPE defects. A multifocal electroretinogram (mfERG) may confirm the diagnosis of HCQ toxicity. The thinning seen on the peripapillary RNFL OCT was not consistent with the clinical examination of the optic disc. Therefore, the diagnosis of glaucoma was excluded.

CWS is the clinical manifestation of focal infarcts of nerve fiber layer. It is localized accumulations of axoplasmic debris in the retinal ganglion cells axon after arteriolar occlusion at the borders of ischemic areas (7). The underlying cause is multiple and include accelerated hypertension. Uncontrolled systemic hypertension leads to various levels of retinal nonperfusion and neuronal loss with subsequent related scotoma (2). Koh et al. reported four cases of nonprogressive localized RNFL and visual field defects after emergence of retinal CWS after followed up for 3 to 19 years. He concluded that it is crucial to consider the visual field results, disc configuration, RNFL defects and fundus findings to decide on the proper diagnosis in patients suspected to have glaucoma (8). On the other hand, Alencar et al. also reported a case of localized RNFL loss with corresponding arcuate defect developing after a retinal CWS in a patient with diabetes and hypertension (9). Chaum et al. also reported a similar case whereby a large idiopathic peripapillary CWS causing nerve fiber bundle visual field defect and an afferent pupillary defect that remained even after the CWS had disappeared (10). Our patient had arcuate scotoma which became less dense throughout follow up. This finding was not reported in the previous the case reports.

\section{Conclusion}

In this case, non-progressive visual field defect secondary to previous hypertensive retinopathy was noted during regular ophthalmology screening for hydroxychloroquine retinopathy. This non-progressive visual field defect may occur after the appearance of CWS in hypertensive retinopathy and it should not be overlooked when diagnosing glaucoma or hydroxychloroquine toxicity.

\section{References}

1. D'Cruz DP, Khamashta MA, Hughes GR. Systemic lupus erythematosus. Lancet. 2007;369(9561):587-96.

2. Schubert HD, Atebara NH, Kaiser RS et al. In: 2014-2015 Basic and clinical science course (BCSC) Section 12: Retina and vitreous. Beach Street, San Francisco, CA: American Academy of Ophthalmology, 2014:113-4.

3. Alarcon GS, McGwin G, Bertoli AM, et al. Effect of hydroxychloroquine on the survival of patients with systemic lupus erythematosus: data from LUMINA, a multiethnic US cohort (LUMINA L). Ann Rheum Dis. 2007;66(9):1168-72. 
4. Marmor MF. Comparison of screening procedures in hydroxychloroquine toxicity. Arch Ophthalmol. 2012;130(4):461-9.

5. Marmor MF, Hu J. Effect of disease stage on progression of hydroxychloroquine retinopathy. JAMA Ophthalmol. 2014;132(9):1105-12.

6. Marmor MF, Kellner U, Lai TY, Melles RB, Mieler WF. Recommendations on screening for chloroquine and hydroxychloroquine retinopathy (2016 revision). Ophthalmology. 2016;123(6):1386-94.

7. Schmidt D. The mystery of cotton-wool spots: a review of recent and historical descriptions. Eur J Med Res 2008;13(6):231-66.
8. Koh JW, Park KH, Kim MS, Kim JM. Localized retinal nerve fiber layer defects associated with cotton wool spots. Jpn J Ophthalmol. 2010;54(4):296-9.

9. Alencar LM, Medeiros FA, Weinreb R. Progressive localized retinal nerve fiber layer loss following a retinal cotton wool spot. Seminars in Ophthalmology. 2007;22(2):103-4.

10. Chaum E, Drewry RD, Ware GT, Charles S. Nerve fiber bundle visual field defect resulting from a giant peripapillary cotton-wool spot. Journal of Neuro-ophthalmology. 2011;21(4):276-7. 